\title{
Electron localization in a two-channel tight-binding model with correlated disorder
}

\author{
V. M. K. Bagci and A. A. Krokhin \\ Department of Physics, University of North Texas, P.O. Box 311427, Denton, Texas 76203, USA
}

(Received 10 May 2007; published 5 October 2007)

\begin{abstract}
We calculate the localization length in a two-channel tight-binding model for correlated disordered site potential. Both intra- and interchannel correlations are taken into account. The localization length is obtained in quadratic approximation by expanding the two-channel conductance over weak disorder. The result is applied to a simple two-stranded model of DNA molecule and it is shown that a strong pair coupling between the basic nucleotides in the strands is not sufficient to delocalize electronic states.
\end{abstract}

DOI: 10.1103/PhysRevB.76.134202

PACS number(s): 72.15.Rn, 72.20.Ee, 87.14.Gg

\section{INTRODUCTION}

A random one-dimensional potential localizes a quantum particle even if the amplitude of the fluctuations is very weak. This fundamental property of a linear random medium is characterized by the localization length $l_{c}$, which gives the scaling for the transmission coefficient through random linear wire of length $L, T(L) \propto \exp \left(-L / l_{c}\right)$. Qualitative characteristics of the transport properties are usually studied in the tight-binding model. ${ }^{1}$ This model is represented by a sequence of potential sites with on-site energies $\varepsilon_{n}$ and constant amplitude of hopping between the neighboring sites $t$. The quantum states in this chain are obtained from the discrete Schrodinger equation,

$$
t\left(\phi_{n+1}+\phi_{n-1}\right)=\left(E-\varepsilon_{n}\right) \phi_{n} .
$$

The strength of the fluctuations is measured by the variance $\varepsilon_{0}^{2}=\left\langle\varepsilon_{n}^{2}\right\rangle$. Since localization occurs for arbitrary weak potential, the case of weak disorder, $\varepsilon_{0} \ll t$, is of a general interest. In this case, the inverse localization length can be calculated in Born approximation and for an uncorrelated potential, $\left\langle\varepsilon_{i} \varepsilon_{k}\right\rangle=\varepsilon_{0}^{2} \delta_{i k}$, it reads $^{2}$

$$
l_{0}^{-1}(E)=\frac{\varepsilon_{0}^{2}}{8\left(1-E^{2} / 4 t^{2}\right)} .
$$

Equation (2) is the first term of the expansion over the powers of disorder, $\varepsilon_{0}^{2}$. The validity of Born approximation suggests that a localized state covers many sites, i.e., $l_{0}(E) \gg 1$; therefore, it is invalid in the vicinity of the band edges, $|E / 2 t| \rightarrow 1$. Here, more accurate expansion over $\varepsilon_{0}$ leads to unusual scaling, $l_{0}^{-1}(E) \propto \varepsilon_{0}^{2 / 3}$. ${ }^{3}$ This "anomalous" behavior of the localization length is accompanied by violation of the scaling hypothesis. ${ }^{4}$ In what follows, we consider the interval of energies in Eq. (2) where the standard Born approximation remains valid.

Correlations in the random sequence of the site energies $\varepsilon_{n}$ strongly affect the interference pattern between the forward and backward scattered waves. In the lowest Born approximation, the localization length is determined by a pair correlation function, $\left\langle\varepsilon_{i} \varepsilon_{k}\right\rangle=\varepsilon_{0}^{2} \xi(i-k)$, only. It was found, using three different variants of perturbation theory, ${ }^{5}$ that in the correlated potential, the localization length is modified and it is given by the following formula:

$$
\gamma(E)=l^{-1}(E)=l_{0}^{-1}(E) \varphi(2 \mu), \quad \varphi(\mu)=1+2 \sum_{k=1}^{\infty} \xi(k) \cos (\mu k) .
$$

Here, the parameter $\mu$ plays the role of Bloch vector, defining the dispersion relation

$$
E=2 t \cos \mu .
$$

Higher-order corrections to Eq. (3) were obtained in Ref. 6. Because of the presence of the Fourier series in the definition of the function $\phi(\mu)$, the Lyapunov exponent [Eq. (3)] may vanish, giving rise to extended states in a one-dimensional disordered system. Extended states may form a discrete set (for short-range correlations) or a continuum of eigenstates. In the latter case, the energy spectrum possesses a mobility edge. Both these peculiarities of the spectra of the systems with short- and long-range correlated disorder have been experimentally observed. ${ }^{7}$

In the majority of publications, the role of correlations has been studied in single-channel tight-binding model. ${ }^{5,8,9}$ Twodimensional or multichannel random systems with correlations got much less attention. Metal-insulator transition in two-dimensional (2D) random lattice with specific symmetry in the distribution of the impurities was predicted in Ref. 10. Recently, the presence of delocalized states in 2D random lattice with long-range correlations has been demonstrated numerically by means of Bloch-like oscillations in dc electric field. ${ }^{11}$ Generalization of the dimer model (short-range correlations) to the two-lag ladder case was done in Ref. 12 and the delocalized state at the band center was obtained. In quasi-one-dimensional models, a continuum of delocalized states may appear for weak long-range correlated disorder. Analytical results for the localization length were obtained for surface ${ }^{13}$ and bulk scatterers ${ }^{14}$ in the limit when interchannel scattering is strongly suppressed, i.e., the electron transport remains effectively one dimensional. This limitation makes it difficult to estimate the localization length in real systems, such as random waveguides or DNA molecule, where inter- and intrachannel scattering amplitudes are of the same order. Moreover, in a DNA molecule, the interchannel coupling is the necessary feature, which originates from the double-helix structure. It was claimed that this coupling may by itself give rise to a continuum of the extended states. ${ }^{15}$ However, this result (based on the numerical simulations of 
the inverse participation ratio) turned out to be erroneous and the long-range correlations are still the necessary condition for the presence of the mobility edge in single- and multichannel random systems. ${ }^{16}$

Here, we consider the two-channel tight-binding model and calculate analytically the localization length for the weakly disordered correlated site potential. We take into account the autocorrelations along each channel and also the cross correlations between the channels. Thus, our approach is valid for the analysis of the electron transport in DNA. In particular, it follows from our general formula for the localization length that the interchannel correlations cannot lead to the extended states, if the site potentials in each channel remain uncorrelated. This confirms the result of Ref. 16.

Electron localization in the multichannel tight-binding model with white-noise potential was recently considered in Refs. 17-19. There, the perturbation theory with respect to weak disorder was developed, using the transfer-matrix approach. $^{22}$ In our calculations, we closely follow the method proposed in Refs. 17-19 and adopt the notations proposed there. However, in the expansion of the transfer (and scattering) matrix, we keep quadratic over weak disorder terms, which contain the effect produced by the correlations. At this point, we modify the perturbation theory of Refs. 17 and 18 , where the linear approximation was sufficient for the case of uncorrelated potential.

\section{TWO-CHANNEL TIGHT-BINDING MODEL}

A generalization of the tight-binding model [Eq. (1)] for the two-channel system is easily done by introducing an index $i$ at the wave function $\phi_{n}$ and the potential energy $\varepsilon_{n}$. This index denotes the channel, $i=1,2$. A term describing the interchannel coupling (with constant hopping parameter $h$ ) is added to the left-hand side. The second equation for the twocomponent wave function $\phi_{i, n}$ is obtained by symmetrizing with respect to the index $i$. Finally, the Schrödinger equation for the two-channel tight-binding model is written as follows:

$$
\begin{aligned}
& t\left(\phi_{1, n+1}+\phi_{1, n-1}\right)+h \phi_{2, n}=\left(E-\varepsilon_{1, n}\right) \phi_{1, n}, \\
& t\left(\phi_{2, n+1}+\phi_{2, n-1}\right)+h \phi_{1, n}=\left(E-\varepsilon_{2, n}\right) \phi_{2, n} .
\end{aligned}
$$

In order to apply the Landauer formula $g=\left(2 e^{2} / h\right) \operatorname{Tr}\left(\hat{t} \hat{t}^{\dagger}\right)$, we consider a two-channel sample of finite length $L=N a$. The spacing $a$ is used as a unit of length. This disordered finitelength sample is connected to the ideal wires, i.e., the site potential $\varepsilon_{n}$ vanishes for $n<1$ and $n>N$. The hopping parameter $t$ enters in Eq. (5) as a natural unit for energy; therefore, in what follows, we take $t=1$ and all the energies are assumed to be normalized to $t$.

A set of equations [Eq. (5)] can be rewritten in matrix form

$$
\left(\begin{array}{c}
\phi_{1, n+1}+\phi_{1, n-1} \\
\phi_{2, n+1}+\phi_{2, n-1}
\end{array}\right)=\left(\begin{array}{cc}
E-\varepsilon_{1, n} & -h \\
-h & E-\varepsilon_{2, n}
\end{array}\right)\left(\begin{array}{l}
\phi_{1, n} \\
\phi_{2, n}
\end{array}\right) .
$$

The two channels remain coupled even in the region of the leads since the nondiagonal elements of the $2 \times 2$ matrix in
Eq. (5) are independent of $n$ and do not vanish. The eigenfunctions for the leads are the plane waves $\exp \left( \pm i \mu_{i} n\right)$ with the wave vectors $\mu_{1}(E)$ and $\mu_{2}(E)$. They are related to the energy by

$$
\begin{aligned}
& 2 \cos \mu_{1}=E-h, \\
& 2 \cos \mu_{2}=E+h .
\end{aligned}
$$

In the region of energies $-2+h<E<2-h$, where both channels are propagating, the quantum states apart from standard degeneracy $E(-\mu)=E(\mu)$ possess additional symmetry with respect to the channel index $i$. Because of this symmetry, there are two different wave vectors, $\mu_{1}$ and $\mu_{2}$, for each value of $E$. In order to develop a perturbation theory, the unperturbed wave functions with definite parity (symmetric and antisymmetric) have to be selected,

$$
\left(\begin{array}{l}
\psi_{1, n} \\
\psi_{2, n}
\end{array}\right)=\frac{1}{\sqrt{2}}\left(\begin{array}{cc}
1 & 1 \\
1 & -1
\end{array}\right)\left(\begin{array}{l}
\phi_{1, n} \\
\phi_{2, n}
\end{array}\right) .
$$

In the $\psi$ basis, the ideal leads are uncoupled and the Schrödinger equation takes the following form:

$$
\begin{aligned}
& \left(\begin{array}{c}
\psi_{1, n+1}+\psi_{1, n-1} \\
\psi_{2, n+1}+\psi_{2, n-1}
\end{array}\right) \\
& \quad=\left(\begin{array}{cc}
E-h-\frac{1}{2}\left(\varepsilon_{1, n}+\varepsilon_{2, n}\right) & \frac{1}{2}\left(\varepsilon_{2, n}-\varepsilon_{1, n}\right) \\
\frac{1}{2}\left(\varepsilon_{2, n}-\varepsilon_{1, n}\right) & E+h-\frac{1}{2}\left(\varepsilon_{1, n}+\varepsilon_{2, n}\right)
\end{array}\right) \\
& \quad \times\left(\begin{array}{c}
\psi_{1, n} \\
\psi_{2, n}
\end{array}\right) .
\end{aligned}
$$

Here, the channels are uncoupled in the region of ideal leads where $\varepsilon_{1, n}=\varepsilon_{2, n}=0$.

The transmission matrix $\hat{t}$ in the Landauer formula is calculated as a product of $N_{L}$ on-site transfer matrices. Due to disorder, these transfer matrices are random. Johnston and Kunz ${ }^{20}$ have shown, using the results of Ref. 21 on the products of random matrices, that the Lyapunov coefficient $\gamma$ is a self-averaging quantity referred to as the inverse localization length,

$$
\gamma(E)=l^{-1}(E)=-\lim _{N_{L} \rightarrow \infty} \frac{1}{2 N_{L}}\left\langle\ln \operatorname{Tr}\left(\hat{t} \hat{t}^{\dagger}\right)\right\rangle
$$

where $\langle\cdots\rangle$ denotes averaging over disorder and $\hat{t}$ is the 2 $\times 2$ transmission matrix.

\section{CALCULATION OF TRANSFER AND SCATTERING MATRIX}

In order to introduce the transfer matrix, we rewrite Eq. (9) in a form of a four-dimensional map, 


$$
\left(\begin{array}{c}
\psi_{1, n+1} \\
\psi_{1, n} \\
\psi_{2, n+1} \\
\psi_{2, n}
\end{array}\right)=\underbrace{\left(\begin{array}{cccc}
E-h-\left(\varepsilon_{1, n}+\varepsilon_{2, n}\right) / 2 & -1 & \left(\varepsilon_{2, n}-\varepsilon_{1, n}\right) / 2 & 0 \\
1 & 0 & 0 & 0 \\
\left(\varepsilon_{2, n}-\varepsilon_{1, n}\right) / 2 & 0 & E+h-\left(\varepsilon_{1, n}+\varepsilon_{2, n}\right) / 2 & -1 \\
0 & 0 & 1 & 0
\end{array}\right)}_{\tilde{x}_{n}}\left(\begin{array}{c}
\psi_{1, n} \\
\psi_{1, n-1} \\
\psi_{2, n} \\
\psi_{2, n-1}
\end{array}\right) .
$$

The matrix $\tilde{X}_{n}$ translates the wave function by one spacing through the site $n$. It is worthwhile to represent this matrix in the basis of unperturbed Bloch waves-the waves propagating in the perfect leads. Since there are no real scatterers in the leads, the translation through any lead site changes only the phase of the wave function, $\psi_{n+1}=e^{ \pm i \mu} \psi_{n}$. The details of this transformation are given in Ref. 17. The translation matrix $\hat{X}_{n}$ for site $n$ in the lead basis is written as follows:

$$
\hat{X}_{n}=X_{0}+X_{n}^{1} \text {. }
$$

The matrix $X_{0}$ is diagonal; it takes into account the accumulation of the phase of the wave function at the translation by the period. The disorder terms, leading to real scattering, are collected in the matrix $X_{n}^{1}$, which is linear over energy fluctuations $\varepsilon_{i, n}$. Equation (12) is valid for arbitrary strength of the random potential. The transfer matrix for the whole sample containing $N_{L}$ sites is a product of $N_{L}$ single-site matrices. In this product, we keep up to quadratic over the random potential terms,

$$
\begin{aligned}
X= & \prod_{n=1}^{N_{L}} \hat{X}_{n} \approx X_{0}^{N_{L}}+\sum_{n=1}^{N_{L}} X_{0}^{N_{L}-n} \cdot X_{n}^{1} \cdot X_{0}^{n-1} \\
& +\sum_{m>n} X_{0}^{N_{L}-m} \cdot X_{m}^{1} \cdot X_{0}^{m-n-1} \cdot X_{n}^{1} \cdot X_{0}^{n-1} .
\end{aligned}
$$

The last two terms are responsible for the scattering and mixing of the modes. From here on, we substitute $L$ instead of $N_{L}$ as the number of sites along the wire for convenience.

Introducing the amplitudes of the Bloch waves at the left and at the right ends of the sample, the matrix $X$ can be represented as the following linear relation between these wave functions:

$$
\left(\begin{array}{c}
a_{1, L}^{+} \\
a_{1, L}^{-} \\
a_{2, L}^{+} \\
a_{2, L}^{-}
\end{array}\right)=X\left(\begin{array}{c}
a_{1,0}^{+} \\
a_{1,0}^{-} \\
a_{2,0}^{+} \\
a_{2,0}^{-}
\end{array}\right) .
$$

In order to calculate the transmission matrix $\hat{t}$, we need to find the scattering matrix $\hat{S}$,

$$
\hat{S}=\left(\begin{array}{cc}
\hat{r}^{-+} & \hat{t}^{--} \\
\hat{t}^{++} & \hat{r}^{+-}
\end{array}\right)
$$

where

$$
\hat{t}^{\mp \mp}=\left(\begin{array}{ll}
t_{11}^{\mp \mp} & t_{12}^{\mp \mp} \\
t_{21}^{\mp \mp} & t_{22}^{\mp \mp}
\end{array}\right), \quad \hat{r}^{ \pm \mp}=\left(\begin{array}{cc}
r_{11}^{ \pm \mp} & r_{12}^{ \pm \mp} \\
r_{21}^{ \pm \mp} & r_{22}^{ \pm \mp}
\end{array}\right) .
$$

Here, $t_{i j}^{++}\left(t_{i j}^{--}\right)$and $r_{i j}^{-+}\left(r_{i j}^{+-}\right)$are the transmission and reflection amplitudes in the channel $i$, provided that there is a unit flux incident from left (right) in the channel $j$. In our case of the two-channel scattering system, the matrix $\hat{S}$ is defined as follows:

$$
\left(\begin{array}{c}
a_{1,0}^{-} \\
a_{2,0}^{-} \\
a_{1, L}^{+} \\
a_{2, L}^{+}
\end{array}\right)=\hat{S}\left(\begin{array}{c}
a_{1,0}^{+} \\
a_{2,0}^{+} \\
a_{1, L}^{-} \\
a_{2, L}^{-}
\end{array}\right) .
$$

Two linear sets [Eqs. (14) and (17)] establish the relations between the elements of the scattering and transfer matrices. It was shown ${ }^{17}$ that the transmission matrix $\hat{t}$ is expressed through the elements of the matrix $X$ as follows:

$$
\begin{gathered}
\hat{t}=\hat{t}^{--}=\frac{1}{\delta}\left(\begin{array}{cc}
X_{44} & -X_{24} \\
-X_{42} & X_{22}
\end{array}\right), \\
\delta=X_{22} X_{44}-X_{24} X_{42} .
\end{gathered}
$$

Now, it is straightforward to expand the conductance [Eq. (10)] over weak disorder and calculate the localization length. This will be done in the next section, separately for the situation when both of the channels are propagating and when one is propagating and the other one is evanescent.

\section{RESULTS FOR LOCALIZATION LENGTH}

\section{A. Two propagating modes}

The expansion of the matrix elements that determine the transmission matrix [Eq. (18)] has the following form:

$$
\begin{aligned}
X_{22}= & e^{-i \mu_{1} L}\left[1+i \sum_{m} a_{m}-\sum_{m>n}\left(1-e^{2 i \mu_{1}(m-n)}\right) a_{m} a_{n}\right. \\
& \left.-\sum_{m>n}\left(e^{i\left(\mu_{1}-\mu_{2}\right)(m-n)}-e^{i\left(\mu_{1}+\mu_{2}\right)(m-n)}\right) c_{m} c_{n}\right], \\
X_{44}= & e^{-i \mu_{2} L}\left[1+i \sum_{m} b_{m}-\sum_{m>n}\left(1-e^{2 i \mu_{2}(m-n)}\right) b_{m} b_{n}\right. \\
& \left.-\sum_{m>n}\left(e^{i\left(\mu_{2}-\mu_{1}\right)(m-n)}-e^{i\left(\mu_{1}+\mu_{2}\right)(m-n)}\right) c_{m} c_{n}\right],
\end{aligned}
$$




$$
\begin{aligned}
& X_{24}=-e^{-i \mu_{1} L}\left[i \sum_{m} e^{i\left(\mu_{1}-\mu_{2}\right) m} c_{m}+O\left(\varepsilon^{2}\right)\right], \\
& X_{42}=-e^{-i \mu_{2} L}\left[i \sum_{m} e^{i\left(\mu_{2}-\mu_{1}\right) m} c_{m}+O\left(\varepsilon^{2}\right)\right] .
\end{aligned}
$$

The elements $X_{24}$ and $X_{42}$ are written in the linear approximation because the quadratic terms there lead to higherorder corrections in the transmission matrix. Substituting these expansions into Eqs. (18) and (10) and performing averaging over disorder, after some algebra, the following formula for the inverse localization length is obtained:

$$
\begin{aligned}
\gamma(E)= & \frac{\varepsilon_{1}^{2}}{64}\left[\frac{\varphi_{11}\left(2 \mu_{1}\right)}{\sin ^{2} \mu_{1}}+\frac{\varphi_{11}\left(2 \mu_{2}\right)}{\sin ^{2} \mu_{2}}+\frac{2 \varphi_{11}\left(\mu_{1}+\mu_{2}\right)}{\sin \mu_{1} \sin \mu_{2}}\right] \\
& +\frac{\varepsilon_{2}^{2}}{64}\left[\frac{\varphi_{22}\left(2 \mu_{2}\right)}{\sin ^{2} \mu_{2}}+\frac{\varphi_{22}\left(2 \mu_{1}\right)}{\sin ^{2} \mu_{1}}+\frac{2 \varphi_{22}\left(\mu_{1}+\mu_{2}\right)}{\sin \mu_{1} \sin \mu_{2}}\right] \\
& +\frac{\varepsilon_{12}}{32}\left[\frac{\varphi_{12}\left(2 \mu_{1}\right)}{\sin ^{2} \mu_{1}}+\frac{\varphi_{12}\left(2 \mu_{2}\right)}{\sin ^{2} \mu_{2}}-\frac{2 \varphi_{12}\left(\mu_{1}+\mu_{2}\right)}{\sin \mu_{1} \sin \mu_{2}}\right] .
\end{aligned}
$$

The structure of this formula for the inverse localization length is similar to the corresponding formula [Eq. (3)] for a single-channel system. The localization of an electron occurs due to elastic backscattering processes in both channels with change of the momentum by $2 \mu_{1}$ and $2 \mu_{2}$ and due to interchannel scattering with change of the momentum by $\mu_{1}$ $+\mu_{2}$. An unperturbed wave, which according to Eq. (8) is either symmetric or antisymmetric, is scattered at three random potentials with variances $\varepsilon_{1}^{2}, \varepsilon_{2}^{2}$, and $\varepsilon_{12}$ and the correlation functions are defined as follows:

$$
\begin{aligned}
\left\langle\varepsilon_{1, n} \varepsilon_{1, n+k}\right\rangle & =\varepsilon_{1}^{2} \xi_{11}(k), \quad\left\langle\varepsilon_{2, n} \varepsilon_{2, n+k}\right\rangle=\varepsilon_{2}^{2} \xi_{22}(k), \quad\left\langle\varepsilon_{1, n} \varepsilon_{2, n+k}\right\rangle \\
& =\varepsilon_{12} \xi_{12}(k) .
\end{aligned}
$$

Here, the mean value $\varepsilon_{12}=\left\langle\varepsilon_{1, n} \varepsilon_{2, n}\right\rangle$ can be either positive or negative, unlike always positive variances $\varepsilon_{1}^{2}$ and $\varepsilon_{2}^{2}$. In the Born approximation, these three potentials give additive contributions to the Lyapunov exponent-a kind of Mattisen rule for the rate of the backscattering processes. The correlations enter through the functions $\varphi_{i j}(\mu)$, which are represented by the Fourier series,

$$
\varphi_{i j}(\mu)=1+2 \sum_{k=1}^{\infty} \xi_{i j}(k) \cos (\mu k) .
$$

As well as in the single-channel case [Eq. (3)], the Fourier coefficient for the $\mu$ th harmonics is the binary correlator of the corresponding potential.

The Lyapunov coefficient [Eq. (21)] is positively defined for all the allowed energies. In the case of uncoupled channels, $h=0$, the dispersion relations [Eq. (7)] are identical, $\mu_{1}=\mu_{2}=\mu$, and the cross-correlation term vanishes. As a result, Eq. (21) is simplified to the following form:

$$
\gamma(E)=\frac{1}{\sin ^{2} \mu}\left[\varepsilon_{1}^{2} \varphi_{11}(2 \mu)+\varepsilon_{2}^{2} \varphi_{22}(2 \mu)\right] .
$$

For identical channels, $\varepsilon_{1}=\varepsilon_{2}$ and $\varphi_{11}(2 \mu)=\varphi_{22}(2 \mu)$, the single-chain case [Eq. (3)] is recovered. Finally, if the potentials in both channels are uncorrelated (white noise), then $\varphi_{i j}(\mu) \equiv 1$ and $\varepsilon_{12}=0$. In this case, Eq. (21) reproduces Heinrichs' result, ${ }^{17}$

$$
\gamma(E)=\frac{\varepsilon_{1}^{2}+\varepsilon_{2}^{2}}{64}\left(\frac{1}{\sin \mu_{1}}+\frac{1}{\sin \mu_{2}}\right)^{2} .
$$

\section{B. One evanescent and one propagating mode}

In the energy regions $-2-h<E<h-2$ and $2-h<E<2$ $+h$, one of the wave numbers $\mu_{i}$ is necessarily a pure imaginary number. The corresponding wave function decays exponentially away from the entering point with decrement $\left|\mu_{i}\right|$. Since the transmission matrix is a relation between the propagating wave modes only, the evanescent modes do not contribute to the conductance of a long sample if $L \gg\left|\mu_{i}\right|^{-1}$.

It was shown in Ref. 18 that in the case of uncorrelated disorder, the evanescent mode term does not contribute to the Lyapunov exponent [Eq. (25)] and has to be omitted. Moreover, the coupling between the propagating and evanescent modes is strongly suppressed. This results in an extra factor of 2 in Eq. (25). In what follows, we demonstrate that this scenario of transition from propagating to evanescent regime remains unchanged in the case of correlated potential.

It is worthwhile to note that in the weak disorder approximation, the formulas for the Lyapunov exponents are invalid in the vicinity of the critical energies $E_{c}= \pm 2 \pm h$, where the transition from propagating to evanescent regime occurs. At these energies, the perturbation $\varepsilon_{i} / \sin \mu_{i} \rightarrow \infty$ and the Born approximation fails even for weak disorder.

Let us consider the energy domain $2-h<E<2+h$ where the second mode is evanescent, $\mu_{2}=i \kappa$. The transfer matrix of the $n$th site, $\hat{X}_{n}$, establishes a linear relation between the wave functions on both sides of this site. Since the evanescent mode does not contribute to the conductance, we are interested only on the elements of the transfer matrix which describe scattering from propagating to propagating mode. For the propagating mode with index 1 , those elements are at the $2 \times 2$ upper left block of the $4 \times 4$ transmission matrix $\hat{X}_{n}$,

$$
\left(\begin{array}{c}
a_{1, n+1}^{+} \\
a_{1, n+1}^{-} \\
\vdots
\end{array}\right)=\left(\begin{array}{ccc}
\hat{X}_{n, 11} & \hat{X}_{n, 12} & \cdots \\
\hat{X}_{n, 21} & \hat{X}_{n, 22} & \cdots \\
\vdots & \vdots & \vdots
\end{array}\right)\left(\begin{array}{c}
a_{1, n+1}^{+} \\
a_{1, n+1}^{-} \\
\vdots
\end{array}\right) .
$$

Now, we can introduce a transfer matrix for the $n$th site, using the basis of the propagating modes only,

$$
\left(\begin{array}{l}
a_{1, n+1}^{+} \\
a_{1, n+1}^{-}
\end{array}\right)=\hat{T}_{n}\left(\begin{array}{l}
a_{1, n}^{+} \\
a_{1, n}^{-}
\end{array}\right), \quad \hat{T}_{n}=\left(\begin{array}{ll}
\hat{X}_{n, 11} & \hat{X}_{n, 12} \\
\hat{X}_{n, 21} & \hat{X}_{n, 22}
\end{array}\right) .
$$

Similar to the transfer matrix $\hat{X}_{n}$, the transfer matrix $\hat{T}_{n}$ has a zero-order diagonal component and linear over the random potential term, 


$$
\hat{T}_{n}=T_{0}+T_{n}^{1}=\left(\begin{array}{cc}
e^{i \mu_{1}} & 0 \\
0 & e^{-i \mu_{1}}
\end{array}\right)+\left(\begin{array}{cc}
i e^{i \mu_{1}} a_{n} & i e^{-i \mu_{1}} \\
-i e^{i \mu_{1}} a_{n} & -i e^{-i \mu_{1}}
\end{array}\right) .
$$

The matrix $\hat{T}_{n}$, being a unitary matrix, conserves the flux. The total transfer matrix $T$ of a sample is a product of all $\hat{T}_{n}$ matrices. Keeping up to quadratic over disorder terms in this product, a formula similar to Eq. (13) is obtained,

$$
\begin{aligned}
T= & \prod_{n=1}^{L} \hat{T}_{n}=T_{0}^{L}+\sum_{n}^{L} T_{0}^{L-n} \cdot T_{n}^{1} \cdot T_{0}^{n-1} \\
& +\sum_{m>n} T_{0}^{L-m} \cdot T_{m}^{1} \cdot T_{0}^{m-n-1} \cdot T_{n}^{1} \cdot T_{0}^{n-1} .
\end{aligned}
$$

In the presence of evanescent mode, the dimension of the scattering matrix is also reduced since now it relates the incoming and outgoing components of the propagating wave only,

$$
\left(\begin{array}{c}
a_{1,0}^{+} \\
a_{1,0}^{-}
\end{array}\right)=T\left(\begin{array}{c}
a_{1, L}^{+} \\
a_{1, L}^{-}
\end{array}\right), \quad\left(\begin{array}{c}
a_{1,0}^{-} \\
a_{1, L}^{+}
\end{array}\right)=\hat{S}\left(\begin{array}{c}
a_{1,0}^{+} \\
a_{1, L}^{-}
\end{array}\right)
$$

The transmission matrix $\hat{t}$, which determines the conductance [Eq. (10)], becomes scalar. It is obtained from the scattering matrix, using Eq. (15),

$$
\hat{S}=\frac{1}{T_{11}}\left(\begin{array}{cc}
T_{21} & 1 \\
1 & -T_{12}
\end{array}\right), \quad t t^{\dagger}=\left|T_{11}\right|^{-2} .
$$

Thus, in the presence of evanescent mode, the conductance (and hence the localization length) is determined by $\left|T_{11}\right|^{2}$. This quantity can be calculated from Eq. (29) and in the quadratic approximation, we get

$$
\begin{aligned}
\left|T_{11}\right|^{2}= & 1+\sum_{m, n} a_{m} a_{n}-2 \sum_{m>n} a_{m} a_{n} \\
& +2 \sum_{m>n} \cos 2 \mu_{1}(m-n) a_{m} a_{n} .
\end{aligned}
$$

Now, expanding $\ln t t^{\dagger}=-\ln \left|T_{11}\right|^{2}$, the following result for the inverse localization length [Eq. (10)] is obtained,

$$
\gamma(E)=\frac{1}{32 \sin ^{2} \mu_{1}}\left[\varepsilon_{1}^{2} \varphi_{11}\left(2 \mu_{1}\right)+\varepsilon_{2}^{2} \varphi_{22}\left(2 \mu_{1}\right)+2 \varepsilon_{12} \varphi_{12}\left(2 \mu_{1}\right)\right] .
$$

In the counterpart region $-2-h<E<-2+h$, the first mode becomes evanescent and the Bloch number $\mu_{1}$ in Eq. (33) is replaced by $\mu_{2}$.

The localization length is a complicated linear functional of the correlation functions $\xi_{i k}(k)$. Analysis of the localization length for different classes of short- and long-range correlations requires separate publication. It is not yet clear what kind of long-range correlations is sufficient for the mobility edge to appear in the spectrum of two-channel system. Here, we give numerical results for the uncorrelated potential and for the exponentially decaying correlation function of the following form:

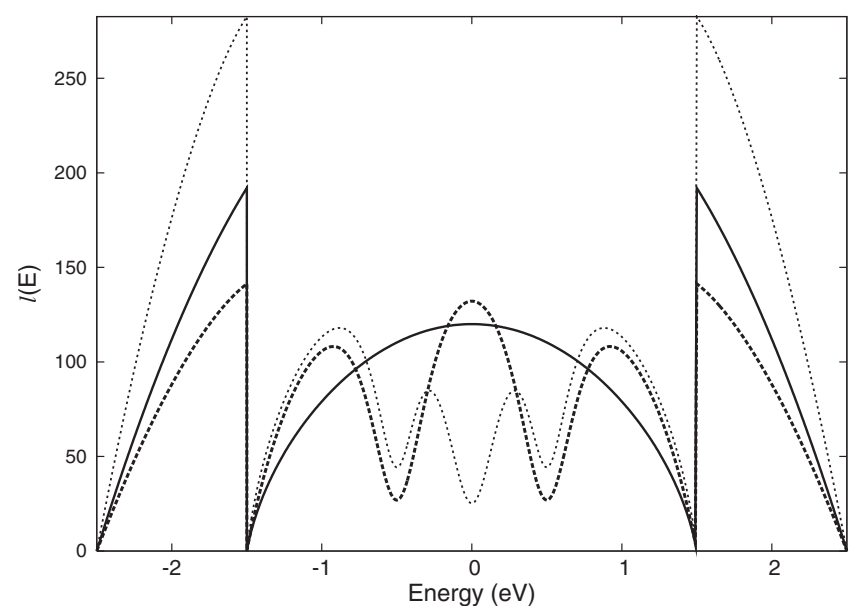

FIG. 1. The localization length vs energy. Solid line is for the uncorrelated (white noise) potential. Dashed-dotted line is for exponential intra- and interchannel correlation functions; all three are given by Eq. (34). Dotted line is for exponential intrachannel correlations and delta-correlated interchannel scattering. The parameters of the model are $h=0.5 \mathrm{eV}, t=1.0 \mathrm{eV}$, and $\left\langle\varepsilon_{1}^{2}\right\rangle=\left\langle\varepsilon_{2}^{2}\right\rangle=\left\langle\varepsilon_{12}^{2}\right\rangle$ $=0.25^{2}$.

$$
\xi_{i j}(k)=(-1)^{k} e^{-\alpha|k|},
$$

where $\alpha$ is the inverse radius of correlations. Here, correlations alternate with anti-correlations. The localization length for this specific correlation function shows oscillatory behavior in the interval of $E$ where both channels are open. (Fig. 1) Unlike this, in the regions where only one of the channels is propagating, the localization length is a monotonic function of energy. There is a discontinuous jump for $l(E)$ at the critical energies $E= \pm(2-h)$ where a transition from propagating to evanescent mode occurs. These discontinuities are clear evidences of the fact that the Born approximation is not valid in the vicinities of the critical points. Extended states do not appear for this class of short-range correlations. They probably may appear if the correlations are of long range, i.e., the correlations decay as a power law.

\section{DISCUSSION}

Equations (21) and (33) are the main results of the paper-they give the localization length in a two-channel system in the whole region of energies. In the case of an uncorrelated potential, the Lyapunov exponent $\gamma(E)$ does not vanish and all the states remain localized ${ }^{17,18}$ as there are in a single-channel system. ${ }^{2}$ Correlations strongly modify the localization length and may give rise to a discrete or continuous set of extended states. ${ }^{5,7-9,13,14}$ Using Eq. (3), it is easy to express the correlator $\xi(k)$ (which plays a role of Fourier coefficient) through the Lyapunov exponent in the singlechannel case,

$$
\xi(k)=\frac{2}{\pi} \int_{0}^{\pi / 2} \gamma[E(\mu)] l_{0}[E(\mu)] \cos (2 k \mu) d \mu .
$$

If the binary correlation function [Eq. (35)] is known, the correlated sequence of the site potential $\varepsilon_{i}$ can be recon- 
structed using the algorithm proposed by Kuhl et al. ${ }^{7}$ Thus, in the single-channel case, the "inverse" scattering problem-reconstruction of the statistical ensemble of the correlated potentials through the localization length $l(E)$ - has a unique solution. It follows from the general properties of the Fourier integrals that if the Lyapunov exponent vanishes within a finite interval of energies, the correlation function decays as a power law, $\xi(k) \propto 1 / k^{p}$. For a sharp mobility edge, the parameter $p=1 .^{5}$

In a two-channel system, the inverse scattering problem is more complicated since there are three correlators, $\xi_{11}(k)$, $\xi_{22}(k)$, and $\xi_{12}(k)$, which have to be reconstructed from the function $\gamma(E)$, given by Eqs. (21) and (22). However, it is clear from the structure of Eqs. (3), (21), and (33) that the power-decaying correlation functions are necessary in order to have a mobility edge. If the correlations are of a short range, only a discrete set of resonant extended states may appear in the spectrum.

In particular, an extended state at the band center $E=0$ was predicted for a two-channel random dimer for some specific parameters of the random potential. ${ }^{12}$ The random dimer is an example of a dichotomous sequence where the on-site potential takes on only two values, e.g., $\varepsilon_{0}$ and $-\varepsilon_{0}$. For all sites, $\varepsilon_{1, n}=\varepsilon_{2, n}$. At a site $n$, the value $\varepsilon_{0}$ or $-\varepsilon_{0}$ emerges with probability of $1 / 2$ and it is repeated at the nearest site $n+1$. Statistical properties of this model are characterized by the mean value $\left\langle\varepsilon_{n}\right\rangle=0$, variances $\left\langle\varepsilon_{1, n}^{2}\right\rangle=\left\langle\varepsilon_{2, n}^{2}\right\rangle=\varepsilon_{0}^{2}, \quad \varepsilon_{12}$ $=\left\langle\varepsilon_{1, n} \varepsilon_{2, n}\right\rangle=\varepsilon_{0}^{2}$, and the correlator $\left\langle\varepsilon_{n} \varepsilon_{n-1}\right\rangle=1 / 2$. By substituting this correlator into Eq. (21), we obtain the inverse localization length for the dimer,

$$
\gamma(E)=\frac{\varepsilon_{0}^{2}}{8}\left(\cot ^{2} \mu_{1}+\cot ^{2} \mu_{2}\right) .
$$

This function does not vanish, if the interchannel hopping parameter $h$ is different from zero, i.e., there is no extended state in a two-channel dimer. Unlike this, in a single-channel dimer there are two extended states, which in the case of weak disorder are situated in the vicinity of the band center $E=0 .{ }^{9}$ Indeed, the Lyapunov exponent [Eq. (36)] vanishes quadratically at a single point $E=0$ if $\mu_{1}=\mu_{2}=\pi / 2$. Equation (36) is valid for weakly disordered potential, when $\varepsilon_{0} \ll 1$; therefore, it does not show the existence of the extended state at $E=0$ for the dimer with $\varepsilon_{0}=1 .^{12}$

Finally, we apply the obtained results to the two-channel system, which models two-stranded DNA molecule. The onsite potential takes on four different values, $\varepsilon_{A}, \varepsilon_{C}, \varepsilon_{T}$, and $\varepsilon_{G}$, associated with four basic nucleotides-adenine, cy- tosine, thymine, and guanine. It is well established that adenine in a strand is always bonded to thymine in the counterpart strand and cytosine - to guanine. In a simple model of DNA, ${ }^{15}$ the four basic nucleotides are evenly represented in a molecule and both strands form uncorrelated sequences of the nucleotides. ${ }^{23}$ In terms of our parameters, it means that $\varepsilon_{12}=\left(\varepsilon_{A} \varepsilon_{T}+\varepsilon_{C} \varepsilon_{G}\right) / 2,\left\langle\varepsilon_{1, n}^{2}\right\rangle=\left\langle\varepsilon_{2, n}^{2}\right\rangle=\left(\varepsilon_{A}^{2}+\varepsilon_{T}^{2}+\varepsilon_{C}^{2}+\varepsilon_{G}^{2}\right) / 4$, and $\left\langle\varepsilon_{1, n+k} \varepsilon_{1, n}\right\rangle=\left\langle\varepsilon_{2, n+k} \varepsilon_{2, n}\right\rangle=0$. Substituting these values into Eq. (21), we get

$$
\begin{aligned}
\gamma(E)= & \frac{1}{128}\left[\left(\varepsilon_{A}+\varepsilon_{T}\right)^{2}+\left(\varepsilon_{C}+\varepsilon_{G}\right)^{2}\right]\left(\frac{1}{\sin ^{2} \mu_{1}}+\frac{1}{\sin ^{2} \mu_{2}}\right) \\
& +\frac{1}{64 \sin \mu_{1} \sin \mu_{2}}\left[\left(\varepsilon_{A}-\varepsilon_{T}\right)^{2}+\left(\varepsilon_{C}-\varepsilon_{G}\right)^{2}\right] .
\end{aligned}
$$

Here, the electron energy $E$ which enters through the dispersion relations [Eq. (7)] and the on-site energies of the nucleotides are counted from the mean site energy $\left\langle\varepsilon_{1, n}\right\rangle$. Since $\sin \mu_{1}$ and $\sin \mu_{2}$ are positive functions $\left(0 \leqslant \mu_{1}, \mu_{2} \leqslant \pi\right)$, the Lyapunov exponent [Eq. (37)] is a sum of two positive quantities, independent of the particular values of the on-site energies. This means that the discussed model of DNA does not allow existence of the extended states. A band of extended states was predicted in Ref. 15, using numerical simulations of the inverse participation ratio. Later, this result has been criticized in Ref. 16 on the basis of group theory arguments. Formula (37) explicitly shows that in a two-channel system, the extended states cannot appear solely due to base pairing A-T, C-G. The base pairing indeed decreases the Lyapunov exponent [Eq. (21)] because of the negative value of the parameter $\varepsilon_{12}$, but this pairing (even being strong) is not enough to give rise to the extended states in the uncorrelated DNA strands. Unlike this, the long-range correlations in a single-stranded model of DNA may lead to a band of extended states. ${ }^{24}$

This discussion shows that the existence of the extended states in DNA is still an open question. Equation (21) may shed light on this problem since this formula takes into account the inter- as well as intrachannel correlations-the effects which were considered separately in the previous studies. The results concerning the existence of the extended states in a double-stranded correlated sequence of nucleotides will be published elsewhere.

\section{ACKNOWLEDGMENT}

This work is supported by the DOE Grant No. DE-FG0206ER46312.
${ }^{1}$ P. W. Anderson, Phys. Rev. 109, 1492 (1958).

${ }^{2}$ D. J. Thouless, Phys. Rev. Lett. 61, 2141 (1988).

${ }^{3}$ B. Derrida and E. Gardner, J. Phys. (Paris) 45, 1283 (1984); F. M. Izrailev, S. Ruffo, and L. Tessieri, J. Phys. A 31, 5263 (1998).

${ }^{4}$ L. I. Deych, A. A. Lisyansky, and B. L. Altshuler, Phys. Rev.
Lett. 84, 2678 (2000); L. I. Deych, M. V. Erementchouk, and A. A. Lisyansky, ibid. 90, 126601 (2003); H. Schomerus and M. Titov, Phys. Rev. B 67, 100201(R) (2003).

${ }^{5}$ J. M. Luck, Phys. Rev. B 39, 5834 (1989); F. M. Izrailev and A. A. Krokhin, Phys. Rev. Lett. 82, 4062 (1999); M. Titov and H. Schomerus, ibid. 95, 126602 (2005). 
${ }^{6}$ L. Tessieri, J. Phys. A 35, 9585 (2002).

${ }^{7}$ V. Bellani, E. Diez, R. Hey, L. Toni, L. Tarricone, G. B. Parravicini, F. Domínguez-Adame, and R. Gómez-Alcalá, Phys. Rev. Lett. 82, 2159 (1999); U. Kuhl, F. M. Izrailev, A. A. Krokhin, and H.-J. Stöckmann, Appl. Phys. Lett. 77, 633 (2000).

${ }^{8}$ J. C. Flores, J. Phys.: Condens. Matter 1, 8471 (1989); F. A. B. F. de Moura and M. L. Lyra, Phys. Rev. Lett. 81, 3735 (1998); J. W. Kantelhardt, S. Russ, A. Bunde, S. Havlin, and I. Webman, ibid. 84, 198 (2000); P. Carpena, P. B. Galvan, P. Ch. Ivanov, and H. E. Stanley, Nature (London) 418, 955 (2002); H. Shima, T. Nomura, and T. Nakayama, Phys. Rev. B 70, 075116 (2004).

${ }^{9}$ D. H. Dunlap, H-L. Wu, and P. W. Phillips, Phys. Rev. Lett. 65, 88 (1990); P. Phillips and H.-L. Wu, Science 252, 1805 (1991).

${ }^{10}$ M. Hilke, Phys. Rev. Lett. 91, 226403 (2003).

${ }^{11}$ F. A. B. F. de Moura, M. L. Lyra, F. Domínguez-Adame, and V. A. Malyshev, J. Phys.: Condens. Matter 19, 056204 (2007).

${ }^{12}$ T. Sedrakyan and A. Ossipov, Phys. Rev. B 70, 214206 (2004).

${ }^{13}$ F. M. Izrailev and N. M. Makarov, Phys. Rev. B 67, 113402 (2003).
${ }^{14}$ L. Tessieri and F. M. Izrailev, J. Phys. A 39, 11717 (2006).

${ }^{15}$ R. A. Caetano and P. A. Schulz, Phys. Rev. Lett. 95, 126601 (2005).

${ }^{16}$ A. Sedrakyan and F. Domínguez-Adame, Phys. Rev. Lett. 96, 059703 (2006); E. Díaz, A. Sedrakyan, D. Sedrakyan, and F. Domínguez-Adame, Phys. Rev. B 75, 014201 (2007).

${ }^{17}$ J. Heinrichs, Phys. Rev. B 66, 155434 (2002).

${ }^{18}$ J. Heinrichs, Phys. Rev. B 68, 155403 (2003).

${ }^{19}$ R. A. Römer and H. Schulz-Baldes, Europhys. Lett. 68, 247 (2004).

${ }^{20}$ R. Johnston and H. Kunz, J. Phys. C 16, 3895 (1983).

${ }^{21}$ V. I. Oseledec, Trans. Mosc. Math. Soc. 19, 197 (1968); V. N. Tutubalin, Theor. Probab. Appl. 13, 65 (1968).

${ }^{22}$ J. B. Pendry, Adv. Phys. 43, 461 (1994).

${ }^{23}$ This statement is not applicable to all DNA's. Many of them exhibit long-range correlations along the strands.

${ }^{24}$ D. Klotsa, R. A. Römer, and M. S. Turner, Biophys. J. 89, 2187 (2005). 